\title{
Why the Milky Way's bulge is not only a bar formed from a cold thin disk
}

\author{
P. Di Matteo ${ }^{1}$, A. Gómez ${ }^{1}$, M. Haywood ${ }^{1}$, F. Combes ${ }^{2}$, M. D. Lehnert ${ }^{3}$, M. Ness ${ }^{5}$, O. N. Snaith ${ }^{6}$, \\ D. Katz ${ }^{1}$, and B. Semelin ${ }^{4,2}$
}

\author{
${ }^{1}$ GEPI, Observatoire de Paris, CNRS, Université Paris Diderot, 5 place Jules Janssen, 92190 Meudon, France \\ e-mail: paola.dimatteo@obspm.fr \\ 2 LERMA, Observatoire de Paris, PSL Research University, CNRS, UMR 8112, 75014 Paris, France \\ 3 Institut d'Astrophysique de Paris, UMR 7095, CNRS, 98bis boulevard Arago, 75014 Paris, France \\ ${ }^{4}$ Sorbonne Universités, UPMC Univ. Paris 6, UMR 8112, LERMA, 75005 Paris, France \\ 5 Max-Planck-Institut für Astronomie, Königstuhl 17, 69117 Heidelberg, Germany \\ ${ }^{6}$ Department of Physics \& Astronomy, University of Alabama, Tuscaloosa, AL 35487, USA
}

Received 24 June 2014 / Accepted 5 November 2014

\section{ABSTRACT}

\begin{abstract}
By analyzing an $N$-body simulation of a bulge formed simply via a bar instability mechanism operating on a kinematically cold stellar disk, and by comparing the results of this analysis with the structural and kinematic properties of the main stellar populations of the Milky Way bulge, we conclude that the bulge of our Galaxy is not a pure stellar bar formed from a pre-existing thin stellar disk, as some studies have recently suggested. On the basis of several arguments emphasized in this paper, we propose that the bulge population that, in the Milky Way, is observed to not be part of the peanut structure corresponds to the old Galactic thick disk, thus implying that the Milky Way is a pure thin+thick disk galaxy, with only a possible limited contribution by a classical bulge.
\end{abstract}

Key words. methods: numerical - Galaxy: bulge - Galaxy: evolution - Galaxy: kinematics and dynamics - Galaxy: abundances

\section{Introduction}

The inner regions of the Milky Way (MW) keep traces of the early phases of the formation of the Galaxy and of its subsequent evolution. From atmospheric chemical abundance studies, we know that $\alpha$-enhanced, metal-poor stars are part of the bulge - the prominent out-of-plane structure characterizing the inner few kpcs of the Galaxy - and this has been interpreted as evidence of its early and rapid enrichment history (McWilliam \& Rich 1994; Zoccali et al. 2006; Fulbright et al. 2007; Lecureur et al. 2007; McWilliam et al. 2008). However, chemical evolution studies also reveal that some of the stars currently in the bulge must have formed in a slower and more quiescent star formation episode, as indicated by the presence of non $\alpha$-enhanced, more metal-rich stars (Bensby et al. 2011; Hill et al. 2011), primarily found closer to the Galactic plane (Ness et al. 2013a).

From stellar kinematics studies, we learn that the velocity ellipsoid of moderate metal-rich $([\mathrm{Fe} / \mathrm{H}]>-0.5 \mathrm{dex})$, non $\alpha$-enhanced stars shows a vertex deviation consistent with those stars supporting a bar-like structure (Zhao et al. 1994; Soto et al. 2007; Babusiaux et al. 2010; Hill et al. 2011). However, such studies also reveal that the most metal-poor, $\alpha$-enhanced stars are not part of this elongated structure, since they have velocity dispersions that are consistent with a kinematically hotter component (Babusiaux et al. 2010; Hill et al. 2011; Uttenthaler et al. 2012; Ness et al. 2013b; Rojas-Arriagada et al. 2014).

Observational studies instead suggest that the MW's bulge does not consist of a single unique component, but is a combination of two or more components or is perhaps a continuum of populations with different chemical and kinematic properties. Even if there is no consensus yet about the origin of the $\alpha$-enhanced, metal-poor stars - classical bulge/old spheroid
(Babusiaux et al. 2010; Gonzalez et al. 2011; Hill et al. 2011; Uttenthaler et al. 2012; Zoccali et al. 2014) or thick disk (Ness et al. 2013b; Di Matteo et al. 2014) - most of the abovecited studies seem to agree on the thin disk origin of the not $\alpha$-enhanced, metal-rich component and its current structure: a bar that went through one or multiple vertical instability events in the past, which led to its current thick, boxy shape, as described by $N$-body models (see, for example, Combes \& Sanders 1981; Athanassoula 2005; Debattista et al. 2006; Martinez-Valpuesta et al. 2006; Ness et al. 2012; Di Matteo et al. 2014).

If observational studies suggest a complex scenario for the formation of the MW's bulge, with the co-existence of multiple or a continuum of components, possibly formed at different times in the Galactic evolution, several $N$-body models have suggested that a pure thin disk instability model can explain the observed characteristics sufficiently well, without the need to add any significant kinematically hot component - classical bulge or thick disk (Shen et al. 2010; Martinez-Valpuesta \& Gerhard 2011, 2013; Kunder et al. 2012; Vàsquez et al. 2013; Gardner et al. 2014; Zoccali et al. 2014). For example, Shen et al. (2010) analyzed an $\mathrm{N}$-body simulation of a disk galaxy that developed a boxy/peanut-shaped bulge and compared it to the stellar kinematics of the bulge region from the BRAVA bulge survey and conclude that "the model fits the BRAVA stellar kinematic data covering the whole bulge strikingly well with no need for a merger-made classical bulge".

Martinez-Valpuesta \& Gerhard (2013) show that pure bar instability models are also able to reproduce vertical metallicity gradients in the Galactic bulge, similar to those observed, provided that the initial disk had a steep enough radial metallicity gradient. They were also able to produce a longitude-latitude 
(hereafter $(l, b))$ metallicity map that is remarkably similar to the one constructed by Gonzalez et al. (2013) from the VVV survey, thus highlighting the result that "a simple model for the Milky Way's boxy bulge", through disk instability, is able to reproduce many of the characteristics observed in the Galactic bulge.

Finally, Zoccali et al. (2014) used the $N$-body model presented in Martinez-Valpuesta \& Gerhard (2011) and compared it with the bulge kinematics from the GIBS survey, noting that no additional kinematically hotter component needs to be added at any latitude to the boxy/peanut-shaped bulge to reproduce the rotation curve and velocity dispersions profiles, thus pointing out that "The very good agreement between this model and the data supports the conclusion presented in Shen et al. (2010)". However, note also that in the same work, Zoccali et al. (2014) cautioned the reader about interpreting the MW's bulge as the result of a pure secular evolution process, recalling, among other things, the observational evidence given at the beginning of this section, about the composite nature of the Galactic bulge.

To conclude, none of the aforementioned $N$-body models has pointed out the necessity to add an additional, kinematically hotter, component to the boxy/peanut-shaped structure, because such a simple scenario was shown to already be able to reproduce all the characteristics considered.

Thus, there is clearly a tension between observations and their interpretation. On the one hand, pure bar instability models fit the global trends observed in the MW's bulge fairly well, without the need to invoke the presence of any additional component - and in some cases, clearly excluding it (Shen et al. 2010) on the basis of this good match. On the other hand observations present a much more complex scenario where the Galactic bulge consists of the co-existence of different populations, with different kinematic and chemical properties.

The aim of this paper is to alleviate this tension, by discussing where and why a pure bar instability mechanism operating on a thin disk fails to represent the complexity of the MW's bulge. By means of a high resolution $N$-body simulation of a thin disk that has undergone a bar instability, we show that this scenario is indeed insufficient when the detailed properties of its main populations are taken into account, despite explaining successfully a number of the properties of the bulge. We show in particular that it is true that $N$-body simulations that suggest a pure thin disk origin for the MW's bulge can reproduce a) the velocity rotation curve and the velocity dispersion profiles of stars in the bulge at different latitudes, as suggested by Shen et al. (2010), Kunder et al. (2012) and Zoccali et al. (2014); b) the vertical metallicity gradient and $(l, b)$-metallicity maps of the bulge which are qualitatively similar to observations, as proposed by Martinez-Valpuesta \& Gerhard (2013) for appropriate initial conditions in the disk, but that these constraints alone are not sufficient to validate a pure bar instability mechanism at the origin of the MW's bulge. Such models indeed lead to bulges with properties that are not compatible with those observed for the MW.

If the MW's bulge uniquely had a thin disk origin and it was only the result of a pure bar instability mechanism originating in a thin stellar disk with a steep enough initial radial metallicity gradient, all bulge stars (from the most metal-rich to the most metal-poor ones) should be part of the boxy/peanut-shaped structure. We show that, if this was the case,

1. all red clump stars in the MW's bulge with $[\mathrm{Fe} / \mathrm{H}]>-1 \mathrm{dex}$ would show a split in the distribution of their $K$ magnitudes, which is not observed (Ness et al. 2013a);
2. the metal-poor population $(-1 \mathrm{dex}<[\mathrm{Fe} / \mathrm{H}] \leq-0.5 \mathrm{dex})$ should be a kinematically warm replica of the more metalrich ones $(-0.5 \mathrm{dex}<[\mathrm{Fe} / \mathrm{H}])$, which is not the case (Ness et al. 2013b).

New observational results that combine the elemental abundances and the kinematics allow us to reassess the evolutionary scenario for the bulge of the MW, and lead us to exclude that the MW's bulge has a pure thin disk origin. This structure is not simply a thick, boxy/peanut-shaped bar formed in a kinematically cold stellar disk and seen edge-on. Its most metalpoor $(-1 \mathrm{dex}<[\mathrm{Fe} / \mathrm{H}] \leq-0.5 \mathrm{dex})$ population does not have a thin disk origin, as recently suggested (Martinez-Valpuesta \& Gerhard 2013), but it is instead associated to an ab initio, kinematically warmer component that - on the basis of several arguments recalled in this paper - we associate to the old Galactic thick disk.

\section{Simulations}

The simulation analyzed in this paper is one of a set of three high resolution simulations with varying bulge-to-disk ratios already described and analyzed in Di Matteo et al. $(2013,2014)$. It consists of an isolated stellar disk, with a $B / D=0.1$ classical bulge $^{1}$ that contains no gas. We chose to present the results for the simulation with $B / D=0.1$ because this ratio is at the suggested upper limit of any classical bulge in the MW (Shen et al. 2010; Kunder et al. 2012; Di Matteo et al. 2014). However, we emphasize that the results obtained for the case with $B / D=0.1$ are identical to those with $B / D=0$, making the two scenarios (pure thin disk versus thin disk + small classical bulge) de facto indistinguishable in the context of the present study. An example of this strong similarity can be appreciated by comparing Figs. 9 and 12 of Di Matteo et al. (2014). Adding a $B / D=0.1$ classical bulge to the simulation has no impact on the overall global kinematic characteristics, neither on the velocity curve nor on the velocity dispersion profiles of the boxy bulge.

The dark halo and the bulge are modeled as Plummer spheres (Binney \& Tremaine 1987). The dark halo has a mass $M_{\mathrm{H}}=$ $1.02 \times 10^{11} M_{\odot}$ and a characteristic radius $r_{\mathrm{H}}=10 \mathrm{kpc}$. The bulge has a mass $M_{\mathrm{B}}=9 \times 10^{9} M_{\odot}$ and characteristic radius $r_{\mathrm{B}}=1.3 \mathrm{kpc}$. The stellar disk follows a Miyamoto-Nagai density profile (Binney \& Tremaine 1987) with mass $M_{*}=9 \times 10^{10} M_{\odot}$, and vertical and radial scale lengths are given by $h_{*}=0.5 \mathrm{kpc}$ and $a_{*}=4 \mathrm{kpc}$, respectively. The initial disk size is $13 \mathrm{kpc}$, and the Toomre parameter is set equal to $Q=1.8$. The galaxy is represented by $N_{\text {tot }}=30720000$ particles redistributed among dark matter $\left(N_{\mathrm{H}}=10240000\right)$ and stars $\left(N_{\text {stars }}=20480000\right)$. To initialize particle velocities, we adopted the method described in Hernquist (1993). A Tree-SPH code (Semelin \& Combes 2002) was used to run the simulations. A Plummer potential was used to soften gravity on scales smaller than $\epsilon=50 \mathrm{pc}$. The equations of motion are integrated over $4 \mathrm{Gyr}$, using a leapfrog algorithm with a fixed time step of $\Delta t=2.5 \times 10^{4} \mathrm{yr}$.

\section{Results}

In what follows, the $N$-body model described in Sect. 2 and extensively studied in Di Matteo et al. $(2013,2014)$ has been

\footnotetext{
1 In the following, by classical bulge we mean a spheroidal component, not formed by disk instabilities, but rather through mergers or some dissipative collapse at early phases of the galaxy formation.
} 

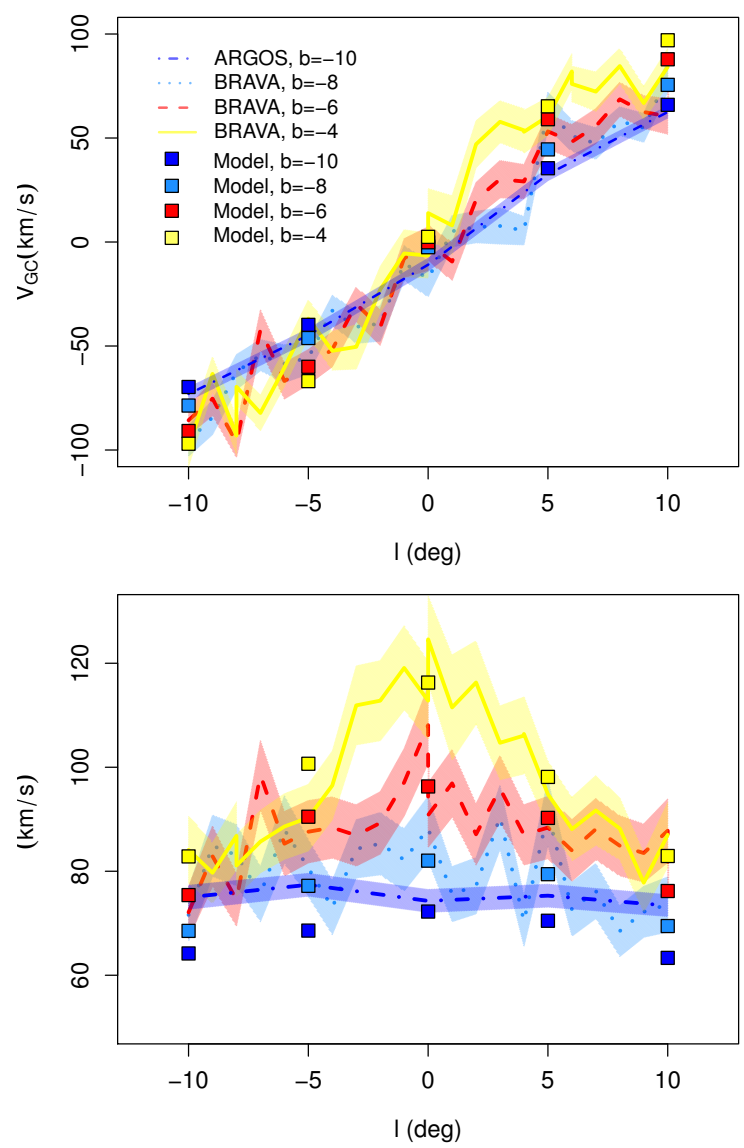

Fig. 1. Rotation curve (top panel) and velocity dispersions (bottom panel) of the stars of the $N$-body models at $|x| \leq 2.5 \mathrm{kpc}$ and $|y| \leq 3 \mathrm{kpc}$ from the galaxy center. Four different latitudes are shown for the modeled galaxy: $b=-4^{\circ}$ (yellow squares), $b=-6^{\circ}$ (red squares), $b=-8^{\circ}$ (pale blue squares), $b=-10^{\circ}$ (dark blue squares). For comparison, BRAVA fields at $b=-4^{\circ}$ (yellow, solid curve), $b=-6^{\circ}$ (red, dashed curve), and $b=-8^{\circ}$ (pale blue, dotted curve), and ARGOS fields at $b=-10^{\circ}$ (dark blue, dash-dotted curve) are also given. The thickness of the curves corresponds to the $\pm 1 \sigma$ error in the observational data.

rescaled to match the MW bar size and bulge velocities ${ }^{2}$. To this aim, we have divided the positions by a factor of about 2 to have a bar length of about $3.5 \mathrm{kpc}$, the corotation at $4.5 \mathrm{kpc}$ and the OLR at $7.5 \mathrm{kpc}$. With this rescaling, the Sun's position is $(0 .,-8 ., 0.) \mathrm{kpc}$. Velocities have been divided accordingly by a factor $\sqrt{2}$, so as to match the rotation curve of the MW's bulge, as deduced by radial velocities measurements (see Kunder et al. 2012; Ness et al. 2013b, and Fig. 1). The bar is then observed at an angle of 20 degrees with respect to the Sun-Galaxy center direction (see, for example, Bissantz \& Gerhard 2002).

\subsection{Successes of a pure thin-disk bar instability scenario}

The rotation curve and velocity dispersion of the final model, as deduced by radial velocity measurements, are shown in Fig. 1, together with data from the ARGOS survey for the fields at $b=-10^{\circ}$ (see Ness et al. 2013b) and from the BRAVA survey for the fields at $b=-4^{\circ},-6^{\circ},-8^{\circ}$ (Kunder et al. 2012). The simulation clearly reproduces the overall kinematic trends found for the MW's bulge, namely the nearly independence of the rotation

\footnotetext{
2 The rescaling is possible because gas is not included in the simulations.
}

curve with latitude ${ }^{3}$ (i.e., cylindrical rotation) and the decrease and flattening of the velocity dispersions with vertical distance from the plane. Thus, in agreement with previous results (Shen et al. 2010; Kunder et al. 2012; Gardner et al. 2014; Zoccali et al. 2014), this $N$-body model supports the finding that a pure bar instability scenario operating on a thin disk can fit the overall kinematics of the MW's bulge, without the need of adding a massive kinematically hotter component (a classical bulge with $B / D>0.1$ or a thick disk) to explain the observed overall kinematic trends.

Thin disk instability scenarios for the formation of the MW's bulge have been shown also to be successful in generating vertical metallicity gradients and metallicity maps consisting of observations. In particular, Martinez-Valpuesta \& Gerhard (2013) have shown that stars in a bar can keep the memory of their initial conditions in such a way that an initial (i.e., before bar formation) radial metallicity gradient in the thin stellar disk can be translated into a vertical gradient in the bulge. An initial radial gradient in the thin disk that is sufficiently steep ${ }^{4}$ ( $-0.4 \mathrm{dex} / \mathrm{kpc}$, as in Martinez-Valpuesta \& Gerhard 2013) would reflect on a vertical gradient along the bulge minor axis similar to the one observed (Zoccali et al. 2008; Gonzalez et al. 2011, 2013). In Fig. 2, we present the $(l, b)$-mean metallicity maps of our simulated galaxy.

The initial radial metallicity profile assigned to the thin stellar disk before bar formation is $[\mathrm{Fe} / \mathrm{H}]=[\mathrm{Fe} / \mathrm{H}]_{0}+\alpha R$, with $\alpha=-0.4 \mathrm{dex} / \mathrm{kpc}, R$ the distance in the plane from the galaxy center, and $[\mathrm{Fe} / \mathrm{H}]_{0}=0.5$ dex the metallicity at $R=0$. For the classical bulge, we adopt a Gaussian metallicity distribution, with a mean at -0.4 dex, which is the typical metallicity of a spheroid of that mass (see Gallazzi et al. 2005; Lee et al. 2008; Thomas et al. 2010), and a dispersion of 0.3 dex. As in Martinez-Valpuesta \& Gerhard (2013), 1) the metallicity maps show a characteristic peanut shape, with the major axis of the peanut elongated parallel to the minor axis of the stellar bar, and 2) a vertical gradient ( $-0.04 \mathrm{dex} / \mathrm{deg}$ in our model, similar to the estimates given in Gonzalez et al. 2013) can be generated in the bulge. These findings are a consequence of the mapping of thin disk stars into the bulge which has been extensively discussed in Di Matteo et al. (2014): stars born in the innermost galaxy regions (where the metallicity attains the highest values, according to the assumed initial conditions) remain mostly confined there, while stars coming from the outer regions of the bar and beyond (up to the bar OLR) are primarily found at higher latitudes (see, e.g., Fig. 7 in Di Matteo et al. 2014). The presence of a small classical bulge $(\sim 10 \%)$ does not have a significant impact on the metallicity map shown in Fig. 2, since the stellar distribution in the boxy bulge is dominated, at all latitudes and longitudes, by stars originating in the disk (see, for example, the top panel of Fig. 11 in Di Matteo et al. 2014).

The ability of pure bar instability processes, originating in the thin disk, to reproduce the global kinematic and chemical

\footnotetext{
3 A mild velocity gradient with latitude is found for stars at positive or negative longitudes, that is with $|l|>0$. For example, at $l=-10^{\circ}$, the velocity gradient is of about $6 \mathrm{~km} \mathrm{~s}^{-1} \mathrm{deg}^{-1}$, stars at $b=-10^{\circ}$ having a rotational velocity that is about $35 \mathrm{~km} \mathrm{~s}^{-1}$ lower than those of stars at $b=-4^{\circ}$. Similar behavior is also found in observational data; see for example Ness et al. (2013b).

4 The pure thin disk scenario requires that the initial radial metallicity gradient is steep at all radii. The adoption of a shallower gradient at radii greater than the bulge size, as done by Bekki \& Tsujimoto (2011b), can lead to a final vertical gradient that is significantly weaker than observed in the MW bulge. We refer the reader to the discussion in Martinez-Valpuesta \& Gerhard (2013) on this point.
} 


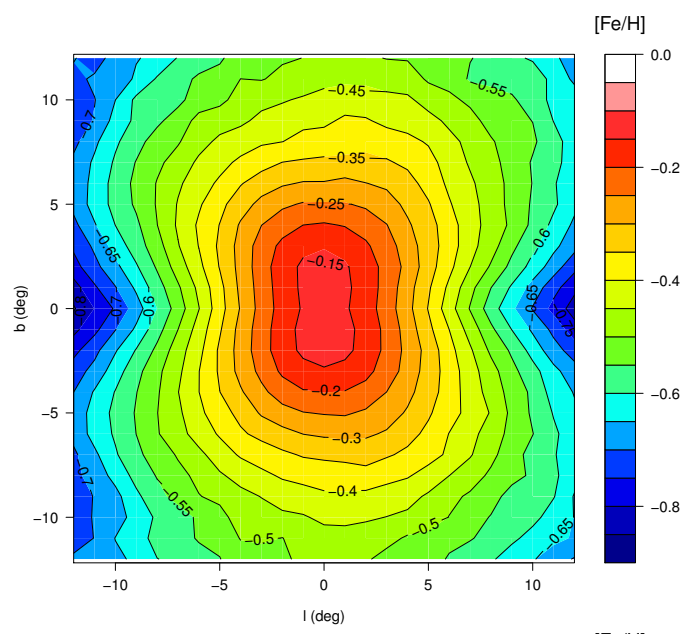

$[\mathrm{Fe} / \mathrm{H}]$

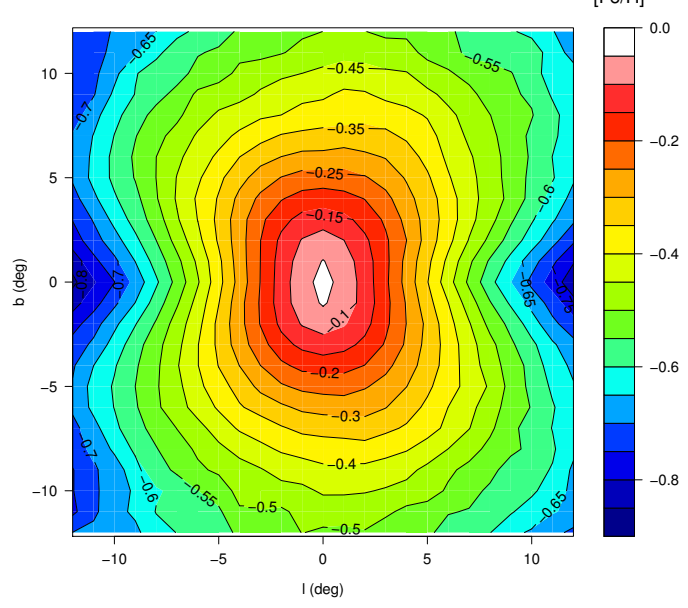

Fig. 2. Metallicity maps of the simulated galaxy in Galactic coordinates. Top panel: disk and classical bulge stars are included; bottom panel: disk stars are only shown (see text for the choice of the initial metallicity of disk and classical bulge stars). In both panels, only stars with $|x| \leq$ $2.5 \mathrm{kpc}$ and $|y| \leq 3 \mathrm{kpc}$ from the galaxy center have been selected. Some contour levels for the metallicity maps are also reported with values given in the maps.

characteristics of the MW's bulge may be considered sufficient to rule out the presence of other components in the inner regions of the Galaxy. In this respect, it seems there is no need to invoke the presence of a massive classical bulge or a thick disk to reproduce the main trends observed. It would be enough that at the time of bar formation, the MW thin disk had a steep radial metallicity gradient accompanied by a positive radial gradient of $[\alpha / \mathrm{Fe}]$ values. If these initial conditions were in place, after the buckling instability, the metal poor, $\alpha$-enriched stars would have been mapped at high latitudes in the bulge, while the most metal-rich and non $\alpha$-enhanced would have stayed preferentially confined closer to the Galaxy midplane, giving rise to the observed vertical metallicity and $[\alpha / \mathrm{Fe}]$ gradients. In Di Matteo et al. (2014), we already commented on the weakness of such a scenario. This would imply that the typical metallicity of the MW disk at 4-5 kpc from the Galaxy center was between -1 and -1.5 dex, and such low metallicities are never reached in the MW thin disk (see, for example, Fuhrmann 1999; Bovy et al. 2012; Haywood et al. 2013), but are typical of the metal-poor tail of the thick disk and of the MW halo (see, among others, Beers \& Sommer-Larsen 1995; Reddy \& Lambert 2008; Nissen \& Schuster 2010). Here we are interested in exploring the consequences that such a scenario would have on the spatial redistribution of stars and on their kinematics, as a function of their chemistry, and showing that the characteristics it would imply do not agree with what we know about the MW's bulge components.

\subsection{Failures of a pure thin-disk bar instability scenario}

The first consequence of a pure thin-disk bar instability scenario for the MW's bulge is that all stars in the bulge fields should be part of the boxy/peanut-shaped structure, independent on their metallicities. For example, as Martinez-Valpuesta \& Gerhard (2013) point out, "the lower limit to the metallicity in the bulge fields would be set by the outer boundary of the part of the disk which participates in the instability".

To quantify the implications of this scenario, we show the distribution of the apparent magnitude of red clump stars in Fig. 3 , as deduced from the $N$-body model, at different latitudes along the bulge minor axis. In producing this distribution, we adopted an absolute magnitude for the clump stars of $M_{K}=-1.61$ (Alves 2000), which gives the minimum of the split red clump at $K=12.9$.

This distribution is particularly suitable to understanding the underlying morphology. The density of a peanut-shaped bar indeed shows some depression in the center, on its minor axis, a depression that is more accentuated at higher latitudes. On the line of sight, this produces a bimodal distribution, or a split, in the apparent $K$-magnitude of the red clump.

In Fig. 3 , the distribution of $K$ magnitudes at different latitudes is shown for stars in the metallicity ranges: $[\mathrm{Fe} / \mathrm{H}]>$ $0 \mathrm{dex},-0.5 \operatorname{dex}<[\mathrm{Fe} / \mathrm{H}] \leq 0 \mathrm{dex},-1 \mathrm{dex}<[\mathrm{Fe} / \mathrm{H}] \leq-0.5 \mathrm{dex}$, corresponding respectively to populations $\mathrm{A}, \mathrm{B}$, and $\mathrm{C}$, as defined by Ness et al. (2013a). As in the previous section, the initial radial metallicity gradient is $\alpha=-0.4 \mathrm{dex} / \mathrm{kpc}$, and the classical bulge has a median metallicity of -0.4 dex and a dispersion of 0.3 dex. Figure 3 shows unequivocally that if the MW's bulge was the result of a pure bar instability mechanism originating in the thin disk, all stars in the bulge should be part of the peanut structure; in particular also those with $-1 \mathrm{dex}<$ $[\mathrm{Fe} / \mathrm{H}] \leq-0.5$ dex should show the split in the distribution of $K$-magnitudes. But this clearly idisagrees with the results presented by Ness et al. (2013a) - and reported in Fig. 3 - who observationally found the split only for red clump stars of higher metallicities. Here lies the failure of the pure thin disk/bar instability model: on the one hand, for the scenario to be successful, one would require metal-poor, $\alpha$-enriched stars to be present in the thin disk before the formation of the bar, in order to participate in the bar instability, be scattered at high vertical distances from the Galactic plane, and as a consequence, constitute the low metallicity part of the metallicity distribution function; on the other hand, the natural and inevitable consequence of such a hypothesis is that those stars should be part of the peanut structure, while they are not, as, for example, the distribution of $K$-magnitudes deduced by the ARGOS survey shows. Even adding the contribution of a low mass classical bulge with $B / D=0.1$, which has been suggested as the upper limit of any classical bulge in the MW (Shen et al. 2010; Di Matteo et al. 2014), the result is unchanged (cf. left and right columns, Fig. 3). All components, from A to C, would be involved in the peanut and show, as a consequence, a split in the distribution of red clump stars. In agreement with the findings of Saha et al. (2012, 2013), such a low mass classical bulge, initially no-rotating, would acquire some angular momentum during the secular evolution (see Fig. 12 in Di Matteo et al. 2014), but - as we checked - would not show any split 
P. Di Matteo et al.: Why the Milky Way's bulge is not only a bar formed from a cold thin disk
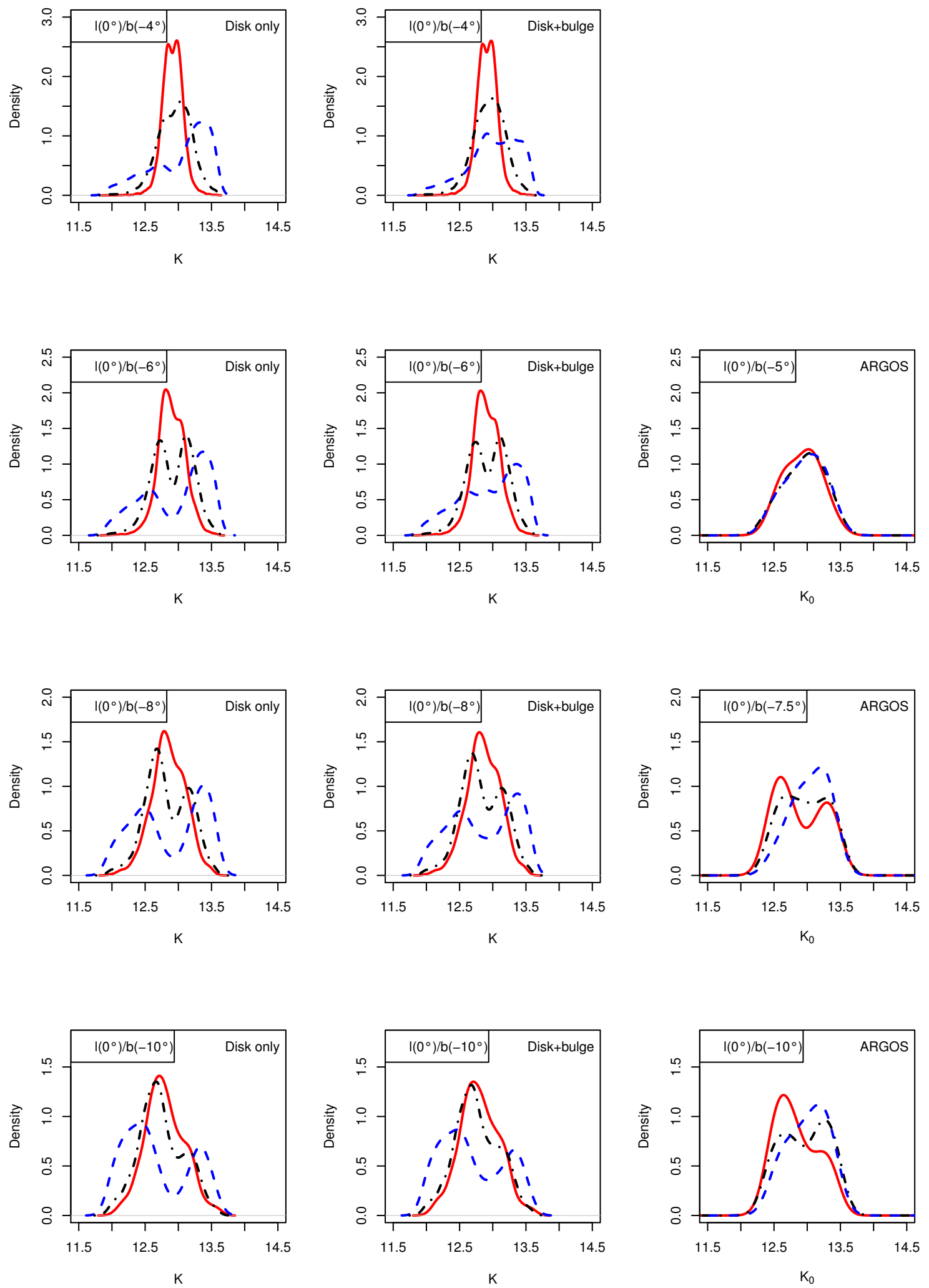

Fig. 3. Left and middle columns: $K$-band magnitude distributions of red clump stars in the modeled galaxy at different latitudes along the bulge minor axis. An initial radial metallicity profile $[\mathrm{Fe} / \mathrm{H}]=0.5-0.4 R$ in the disk is assumed, as in Martinez-Valpuesta \& Gerhard (2013). Three different metallicity bins are then shown: $[\mathrm{Fe} / \mathrm{H}]>0$ (red, solid curve), $-0.5<[\mathrm{Fe} / \mathrm{H}] \leq 0$ (black, dash-dotted curve), $-1<[\mathrm{Fe} / \mathrm{H}] \leq-0.5$ (blue, dashed curve), similar to populations A, B, and C, as defined by Ness et al. (2013a). Disk stars only are shown in the left panel, while all (i.e., disk and classical bulge) stars are shown in the right panel. In all panels, stars with $(l, b)$ in the interval $\left(l_{0}, b_{0}\right) \pm(\Delta l, \Delta b)$ are shown, with $\Delta l=\Delta b=1^{\circ}$ and $\left(l_{0}, b_{0}\right)$ as given in the top left corner. Right column: $K_{0}$-band magnitude distributions of red clump stars at different latitudes along the bulge minor axis from the ARGOS survey. See Ness et al. (2013a) for details. 

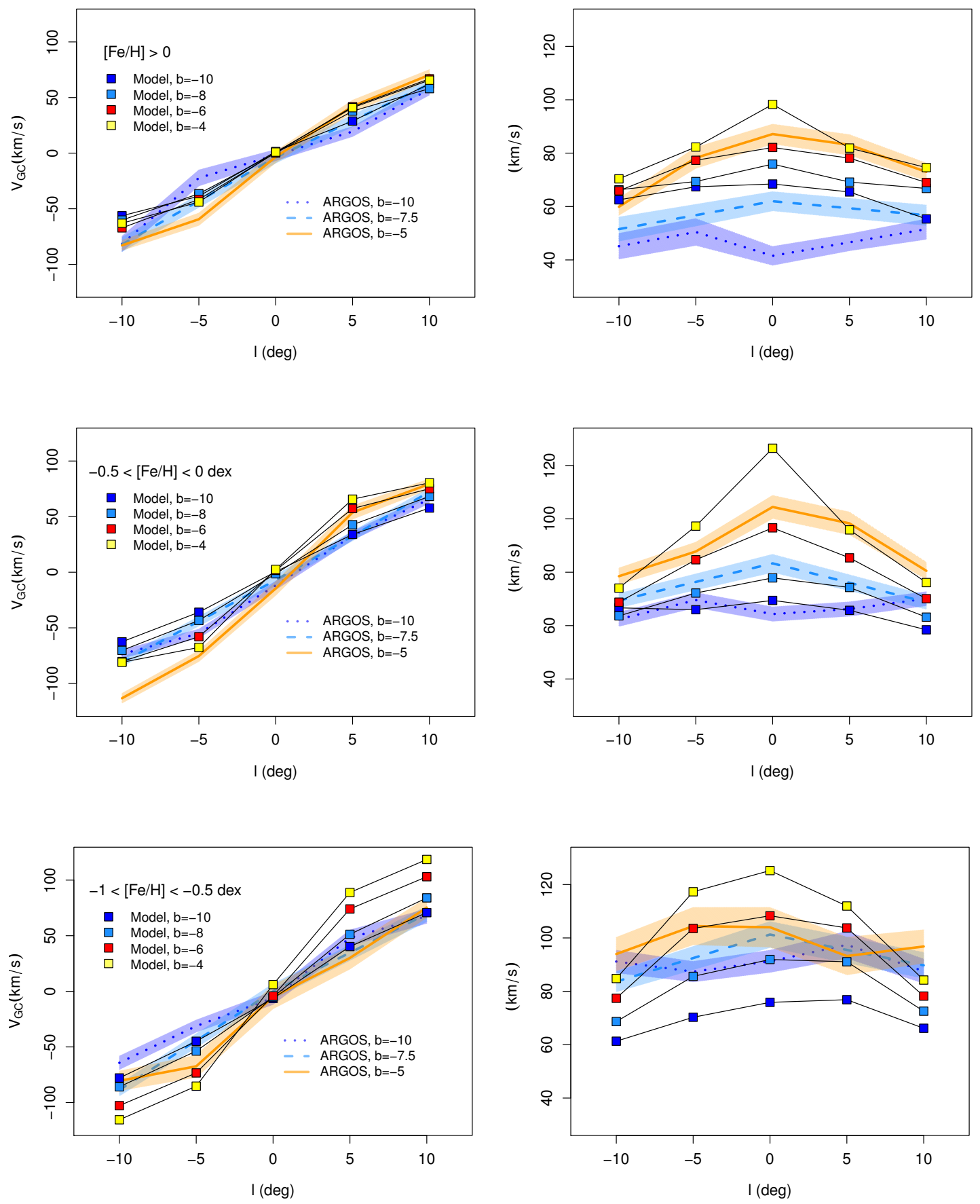

Fig. 4. Rotation curve (left panels) and velocity dispersions (right panels) of a boxy bulge formed from a thin stellar disk (square symbols) compared to ARGOS data (colored curves). In the $N$-body model, only stars at $|x| \leq 2.5 \mathrm{kpc}$ and $|y| \leq 3 \mathrm{kpc}$ from the galaxy center are shown. An initial radial metallicity profile $[\mathrm{Fe} / \mathrm{H}]=0.5-0.4 R$ in the disk is assumed, similar to Martinez-Valpuesta \& Gerhard (2013). Three different metallicity bins are shown, from top to bottom in decreasing $[\mathrm{Fe} / \mathrm{H}]$, corresponding to the populations $\mathrm{A}$, B, and $\mathrm{C}$, as defined by Ness et al (2013a). For each plot, four different latitudes are shown for the modeled galaxy: $b=-4^{\circ}$ (yellow squares), $b=-6^{\circ}$ (red squares), $b=-8^{\circ}$ (pale blue squares), $b=-10^{\circ}$ (dark blue squares). For comparison, ARGOS fields at $b=-5^{\circ}$ (orange, solid curve), $b=-7.5^{\circ}$ (pale blue, dashed curve), $b=-10^{\circ}$ (dark blue, dotted curve) for populations A, B, and $\mathrm{C}$ are also given. The thickness of the curves corresponds to the $\pm 1 \sigma$ error in the observational data.

in the $K$-magnitude distribution, indicating that it does not participate in the bar. Overall, it would only leave very weak signatures in the $K$-distribution, and, coupled with an initial steep disk metallicity gradient, it would not erase the characteristic split of the peanut.

The $K$-magnitude distribution of red clump stars is only an example of the discrepancy with observations that such a bulge formation scenario would imply. Also the detailed kinematic characteristics of a bulge formed only via bar instability in a pre-existing thin stellar disk would not agree with observations. To elucidate this point, in Fig. 4 we show the velocity curves and velocity dispersions profiles that stars in the three different metallicity bins formerly defined would have in such a scenario. Similar to observations, which often lack proper-motion measurements for bulge stars, only the radial component of the velocity is shown in this plot. For comparison, the velocities and 
velocity dispersions observed for populations $\mathrm{A}, \mathrm{B}$, and $\mathrm{C}$, as defined by Ness et al. (2013a), are also shown. If all stars in the bulge MDF had a thin disk origin, according to Fig. 4, the following kinematic trends should be observed:

1. The velocity dispersion would increase with decreasing metallicity, in such a way that population $\mathrm{C}$ would be a warm replica of populations A and B; that is, it should show velocity dispersion profiles similar to those of the populations associated to the bar, but shifted to higher absolute values.

2. The rotational velocity would increase with decreasing metallicity continuously from super-solar metallicities to $[\mathrm{Fe} / \mathrm{H}]=-1 \mathrm{dex}$.

As explained in Di Matteo et al. (2014), the trends at points 1 and 2 are the simple consequence of the differential mapping of a thin disk onto a boxy bulge: the larger the birth radius of stars, the higher their rotational velocity and velocity dispersion.

In Di Matteo et al. (2014), we compared these trends with the results by Ness et al. (2013b) and concluded that the MW's bulge population $\mathrm{B}(-0.5 \mathrm{dex}<[\mathrm{Fe} / \mathrm{H}] \leq 0$ dex $)$ must have on average a more external disk origin than population $\mathrm{A}([\mathrm{Fe} / \mathrm{H}]>$ 0 dex). Indeed, according to the ARGOS observations reported in Fig. 4 and to the discussion in Ness et al. (2013b), population $\mathrm{B}$ shows a higher rotational velocity and similar velocity dispersions profiles (but with higher absolute values) than population $\mathrm{A}$, as would be the case if component $\mathrm{B}$ formed on average farther out from the galaxy center than component A.

However, for the purpose of the present discussion, we want to emphasize that points 1 and 2 also lead us to exclude the idea that the more metal-poor population $\mathrm{C}(-1 \mathrm{dex}<[\mathrm{Fe} / \mathrm{H}] \leq$ -0.5 dex) can have an origin in the old MW thin disk. From Martinez-Valpuesta \& Gerhard (2013) and Sect. 3.1, we have indeed learnt that a steep metallicity radial gradient in the thin disk is necessary before bar formation occurs, in order to reproduce a vertical gradient along the bulge minor axis, as currently observed. Thus in this scenario, the metal poor population $\mathrm{C}$ would initially have been - i.e., before the onset of the bar instability - a thin disk population located farther out from the center than populations A and B. The subsequent bar vertical instability process would have scattered these stars to large distances from the plane, leading this component to have kinematic properties similar to those found in Fig. 4 for the most metal-poor stars. That is, in this scenario $\mathrm{C}$ should show a rotational velocity greater than that of populations $\mathrm{A}$ and $\mathrm{B}$, and its velocity dispersions would be a simple warm replica of those of the most metal rich populations. But this is excluded by the observational data (Ness et al. 2013b), which shows velocity dispersions that are constant both with longitude and latitude, and a rotational velocity similar to that of populations A and B.

Overall, our results point to the necessity of making use of both the kinematic and elemental abundance information from the various bulge components to understand its origin. While this is obviously true, why is it that most analyses based on $\mathrm{N}$-body simulations have not been able to capture the kinematically warmer component that is apparent in the data? The reason is simple. Among the main components A, B, and $\mathrm{C}$ found in the MW's bulge, most of the numerical models (Shen et al. 2010; Kunder et al. 2012; Ness et al. 2013b; Zoccali et al. 2014), including this work, have essentially been able to only capture component $\mathrm{B}$.

B constitutes the backbone of the MW's bulge (see Ness et al. 2013a) and the bulge's overall kinematic trends are represented well by population B (see Figs. 3 and 6 in Ness et al. 2013b). Thus a pure thin disk $N$-body model that fits the global kinematics of the MW's bulge by means of a single stellar population essentially fits, and captures, the properties of population $\mathrm{B}$ alone $\mathrm{e}^{5}$. It is only by modeling both the peanutshaped components $\mathrm{A}$ and $\mathrm{B}$ that $N$-body simulations can point to the existence of a kinematically warmer component like $\mathrm{C}$. This because including component $\mathrm{A}$ would result in a bulge with global kinematics that are colder than what is generally found in models that reproduce B alone, and, as a consequence, it would be possible and even necessary to accommodate a kinematically warmer component to fit the global trends.

\section{Discussion: The Milky Way as a pure (thin+thick) disk galaxy}

If a pure thin disk/bar instability scenario for the MW's bulge can be rejected, does this imply that our Galaxy is not a pure disk galaxy, as suggested by Shen et al. (2010)? This question is closely related to this second one: what is the nature of the kinematically hotter component $\mathrm{C}$ found in the inner Galactic region, whose origin cannot be related to the thin disk?

The answer to this second question is still somewhat speculative at this stage, but it is worth suggesting that the growing evidence and recent results should inspire us to critically revise our view of the populations and components of the MW. From the work of Bensby et al. (2011) and Bovy et al. (2012), we learn that the $\alpha$-enhanced, metal-poor population of the Galaxy, coincident with the thick disk in the solar vicinity, has a scale length of about $1.8 \mathrm{kpc}$, which is a factor of about 2 less than previous estimates (see, for example, Juric̀ et al. 2008). This finding, coupled with the thin/thick disk local normalization, implies that the thick disk is under-represented at the solar vicinity, and it is instead mostly concentrated toward the inner Galactic regions, where it can become comparable to the thin disk mass (see discussion in Snaith et al. 2014; and also Fuhrmann et al. 2012). The finding that the thick disk mass is comparable to that of the thin disk is not just the result of the simple structural arguments given previously. It finds strong support and independent confirmation in the recent work by Snaith et al. (2014). Snaith et al. modeled the $[\alpha / \mathrm{Fe}]$-age relation recently discovered for disk stars in the solar vicinity (Haywood et al. 2013) with a simple closed-box evolutionary scenario. Snaith et al. showed that: i) the thick disk formed during the most intense phase of star formation in the Galaxy; ii) this phase, which lasted from about 13 to 8-9 Gyr ago, formed as many stars as the subsequent, more quiescent, phase of star formation in the disk, which proceeded from 8-9 Gyr ago to the present epoch during which the thin disk was formed. The findings of Snaith et al. (2014) are in substantial agreement with the independent work of van Dokkum et al. (2013), who on the basis of abundance matching techniques, followed the evolution of "MW-like" progenitors from $z=2.5$ to the present epoch, concluding that these galaxies formed half of their stellar mass before redshift 1 (see Snaith et al. 2014; and also Lehnert et al. 2014). Their results indicate that before $z=1$, these galaxies were growing at all radii, with the inner regions growing at the same rate as the outer regions. As

\footnotetext{
5 In our model, this can be appreciated in Figs. 1 and 4: from Fig. 4 it is evident that the modeled population B is the only which satisfactory captures the kinematics of the corresponding observed stellar component (both components with $[\mathrm{Fe} / \mathrm{H}]>0$ and $[\mathrm{Fe} / \mathrm{H}]<-0.5 \mathrm{dex}$ do not capture the kinematics of the corresponding populations $\mathrm{A}$ and $\mathrm{C}$ observed by ARGOS); comparing Fig. 4 with Fig. 1, it is evident that the kinematics of modeled component $\mathrm{B}$ is representative of the global bulge kinematics.
} 
van Dokkum et al. (2013) point out, in these MW-analogues "we do not see high- density naked bulges at $z \sim 2$ around which disks gradually assembled. ... The evolution from $z=2.5$ to $z=1$ is strikingly uniform: the profiles are roughly parallel to one another, and rather than assembling only inside out, the galaxies increase their mass at all radii. This is in marked contrast to more massive galaxies, which form their cores early and exclusively build up their outer parts over this redshift range". The interpretation of the van Dokkum et al. (2013) results, in light of the works by Snaith et al. (2014) and Lehnert et al. (2014), strongly suggest that:

- in these high redshift MW analogs we are witnessing the formation of thick disks;

- these galaxies were not forming any significant classical bulge at those times.

While we refer to Lehnert et al. (2014) for a detailed discussion of the properties of the MW at high redshift, we emphasize here that, if confirmed, this formation and evolutionary scenario would be substantially different from those suggesting that the old, metal-poor, $\alpha$-enhanced population of the MW's bulge can be explained by a classical bulge or old spheroid (Babusiaux et al. 2010; Gonzalez et al. 2011; Hill et al. 2011; Uttenthaler et al. 2012; Zoccali et al. 2014). Recently, for example, Zoccali et al. (2014) have suggested that the oldest component of MW's bulge resembles a low mass, early-type galaxy with properties similar to those of current early-type galaxies, as described by the SAURON and ATLAS3D samples (Bacon et al. 2001; Cappellari et al. 2011). While it is true that an old thick disk can resemble a fast-rotating early-type system in many aspects, the classical bulge/old spheroid scenario would imply that what we observe in the MW inner regions cannot be explained essentially on the basis of the known Galactic populations in the solar vicinity (i.e. thin and thick disks and only very marginally by a stellar halo), but instead requires including an additional kinematically hot component, which is not negligible in terms of mass, in the central Galactic regions.

The disagreement between the two scenarios is thus much more than a simple semantic difference. Even claiming (Zoccali et al. 2014) an instability mechanism via clump formation and coalescence in the galaxy center (Noguchi 1999; Immeli et al. 2004; Elmegreen et al. 2008) to form the MW's bulge raises some doubts. The most recent models show that this evolutionary channel is inefficient at forming massive $(B / D>10 \%)$ classical bulges in MW-like galaxies (Bournaud et al. 2014). Moreover, $N$-body simulations of classical bulge formation by clumpy instabilities show that these structures are usually slow rotators (see Elmegreen et al. 2008), a property hardly reconcilable with the fast rotation of population C (see Ness et al. 2013b). These considerations, coupled with the fact that the metallicity of component $\mathrm{C}$ is unlikely for a classical bulge of $\sim 10^{10} M_{\odot}$ (see discussion in Di Matteo et al. 2014, and references therein), strongly suggest the possibility that the MW's bulge is the result of the simple mapping of the Galactic (thin + thick) disk in the central regions of the Galaxy, with the kinematically coldest part of the disk captured in the bar vertical instability (and thus mapped into populations A and B) and the kinematically hottest component forming population $\mathrm{C}$ (see also the discussion in Ness et al. 2013b). N-body simulations of boxy/peanut-shaped bulges formed in galaxies containing both a thin and a thick stellar disk seem to support this scenario by showing that the strength of the peanut-shaped structure depends on the origin of the stars, with thin disk stars showing a more prominent peanut-shaped bar than stars originating in the thick disk (see Bekki \& Tsujimoto 2011a, their Fig. 10). These (thin+thick) disk models can also reproduce both the observed cylindrical rotation and vertical metallicity gradient of the Galactic bulge reasonably well (Bekki \& Tsujimoto 2011b).

Even if further models are needed to test whether this scenario is able to reproduce the chemo-kinematic trends unraveled by the most recent spectroscopic surveys of the Galactic bulge, we emphasize that currently

1. solar vicinity data (see Fuhrmann et al. 2012; Haywood et al. 2013; Snaith et al. 2014),

2. Galactic kpc-scale observations (Bensby et al. 2011; Bovy et al. 2012) and

3. the redshift evolution of MW-like analogs (van Dokkum et al. 2013),

all seem to imply a substantial role for thick disks in the evolution of MW-like galaxies, a role still underestimated in the current debate on the nature of the MW's bulge populations. The recent findings of strong similarities between the $[\alpha / \mathrm{Fe}]$ versus $[\mathrm{Fe} / \mathrm{H}]$ trends of solar vicinity thick disk stars with the $\alpha$-enhanced, metal-poor population of the bulge (Melèndez et al. 2008; Alves-Brito et al. 2010; Bensby et al. 2011, 2013; Gonzalez et al. 2011), would be a strong additional support to this scenario if confirmed with larger high resolution spectroscopic samples.

\section{Conclusions}

By analyzing a high resolution, $N$-body simulation of a bulge formed via a simple bar instability mechanism in a thin disk, we have shown that such a scenario is not compatible with the known structural and kinematic properties of the main populations of the Galactic bulge. In particular, we emphasize that global kinematic and metallicity trends alone are not sufficient to constraint the MW's bulge formation scenario. It is only by coupling kinematic and abundances information that $\mathrm{N}$-body models are able to reject a pure thin disk/bar instability process to explain the formation and characteristics of the MW's bulge. Thus, in disagreement with recent suggestions, we conclude that the Milky Way bulge is not simply a bar originating in a kinematically cold stellar disk and seen edge-on. Its components did not all originate in the thin disk.

On the basis of recent observational evidence, recalled in this paper, we suggest that the metal poor, $\alpha$-enhanced population that is present in the bulge, but which is not part of the peanut structure, is the same population as is known at the solar vicinity as the old thick disk.

Acknowledgements. The authors acknowledge the support of the French Agence Nationale de la Recherche (ANR) under contract ANR-10-BLAN-0508 (GalHis project). MN acknowledges funding from the European Research Council under the European Unions Seventh Framework Programme (FP 7) ERC Grant Agreement No. [321035]. Support for ONS was partially provided by NASA through the Hubble Space Telescope Archival Research grant HST-AR12837.01-A from the Space Telescope Science Institute, which is operated by the Association of Universities for Research in Astronomy, Incorporated, under NASA contract NAS5-26555. The authors are grateful to N. Stefanovitch for his technical help. P.D.M. thanks F. Matteucci and C. Morossi for organizating of the stimulating conference on the "Formation and Evolution of the Galactic Bulge" (Sesto, Italy, January 2014), where many of the ideas presented in this paper took shape. PDM is grateful to F. Bournaud for an enriching discussion of the current state of knowledge of clumpy disk galaxies. We thank the referee for a very constructive report, which greatly helped us to clarify our results.

\section{References}

Alves, D. R. 2000, ApJ, 539, 732

Alves-Brito, A., Meléndez, J., Asplund, M., Ramírez, I., \& Yong, D. 2010, A\&A, 513, A35 
Athanassoula, E. 2005, MNRAS, 358, 1477

Babusiaux, C., Gómez, A., Hill, V., et al. 2010, A\&A, 519, A77

Bacon, R., Copin, Y., Monnet, G., et al. 2001, MNRAS, 326, 23

Beers, T. C., \& Sommer-Larsen, J. 1995, ApJS, 96, 175

Bekki, K., \& Tsujimoto, T. 2011a, ApJ, 738, 4

Bekki, K., \& Tsujimoto, T. 2011b, MNRAS, 416, L60

Bensby, T., Alves-Brito, A, Oey, M. S., Yong, D., \& Meléndez, J. 2011, ApJ, 735, L46

Bensby, T., Yee, J. C., Feltzing, S., et al. 2013, A\&A, 549, A147

Binney, J., \& Tremaine, S. 1987, Galactic Dynamics (Princeton: Princeton Univ. Press)

Bissantz, N., \& Gerhard, O. 2002, MNRAS, 330, 591

Bournaud, F., Perret, V., Renaud, F., et al. 2014, ApJ, 780, 57

Bovy, J., Rix, H.-W., Liu, C., et al. 2012, ApJ, 753, 148

Cappellari, M., Emsellem, E., Krajnović, D., et al. 2011, MNRAS, 413, 813

Combes, F., \& Sanders, R. H. 1981, A\&A, 96, 164

Debattista, V. P., Mayer, L., Carollo, C. M., et al. 2006, ApJ, 645, 209

Di Matteo, P., Haywood, M., Combes, F., Semelin, B., \& Snaith, O. 2013, A\&A, 553, A102

Di Matteo, P., Haywood, M., Gómez, A., et al. 2014, A\&A, 567, A122

Elmegreen, B. G., Bournaud, F., \& Elmegreen, D. M. 2008, ApJ, 688, 67

Fuhrmann, K. 1999, Ap\&SS, 265, 265

Fuhrmann, K., Chini, R., Hoffmeister, V. H., \& Bernkopf, J. 2012, MNRAS, 420, 1423

Fulbright, J. P., McWilliam, A., \& Rich, R. M. 2007, ApJ, 661, 1152

Gallazzi, A., Charlot, S., Brinchmann, J., White, S. D. M., \& Tremonti, C. A. 2005, MNRAS, 362, 41

Gardner, E., Debattista, V. P., Robin, A. C., et al. 2014, MNRAS, 438, 3275

Gonzalez, O. A., Rejkuba, M., \& Zoccali, M., et al. 2011, A\&A, 530, A54

Gonzalez, O. A., Rejkuba, M., Zoccali, M., et al. 2013, A\&A, 552, A110

Haywood, M., Di Matteo, P., Lehnert, M. D., Katz, D., \& Gómez, A. 2013, A\&A, 560, A109

Hernquist, L. 1993, ApJS, 86, 389

Hill, V., Lecureur, A., Gómez, A., et al. 2011, A\&A, 534, A80

Immeli, A., Samland, M., Gerhard, O., \& Westera, P. 2004, A\&A, 413, 547

Jurić, M., Ivezić, Z., Brooks, A., et al. 2008, ApJ, 673, 864
Kunder, A., Koch, A., Rich, R. M., et al. 2012, AJ, 143, 57

Lecureur, A., Hill, V., Zoccali, M., et al. 2007, A\&A, 465, 799

Lee, H., Bell, E. F. \& Somerville, R. S. 2008, Low-Metallicity Star Formation: From the First Stars to Dwarf Galaxies, eds. L. K. Hunt, S. Madden, \& R. Schneider (Cambridge: Cambridge Univ. Press), IAU Symp., 255, 100

Lehnert, M. D., Di Matteo, P., Haywood, M., \& Snaith, O. N. 2014, ApJ, 789, L30

Martinez-Valpuesta, I., \& Gerhard, O. 2011, ApJ, 734, L20

Martinez-Valpuesta, I., \& Gerhard, O. 2013, ApJ, 766, L3

Martinez-Valpuesta, I., Shlosman, I., \& Heller, C. 2006, ApJ, 637, 214

McWilliam, A., \& Rich, R. M. 1994, ApJS, 91, 749

McWilliam, A., Matteucci, F., Ballero, S., et al. 2008, AJ, 136, 367

Meléndez, J., Asplund, M., Alves-Brito, A., et al. 2008, A\&A, 484, L21

Ness, M., Freeman, K., Athanassoula, E., et al. 2012, ApJ, 756, 22

Ness, M., Freeman, K., Athanassoula, E., et al. 2013a, MNRAS, 430, 836

Ness, M., Freeman, K., Athanassoula, E., et al. 2013b, MNRAS, 432, 2092

Nissen, P. E., \& Schuster, W. J. 2010, A\&A, 511, L10

Noguchi, M. 1999, ApJ, 514, 77

Reddy, B. E., \& Lambert, D. L. 2008, MNRAS, 391, 95

Rojas-Arriagada, A., Recio-Blanco, A., Hill, V., et al. 2014, A\&A, 569, A103

Saha, K., \& Gerhard, O. 2013, MNRAS, 430, 2039

Saha, K., Martinez-Valpuesta, I., \& Gerhard, O. 2012, MNRAS, 421, 333

Semelin, B., \& Combes, F. 2002, A\&A, 388, 826

Shen, J., Rich, R. M., Kormendy, J., et al. 2010, ApJ, 720, L72

Snaith, O., Haywood, M., Di Matteo, P., et al. 2014, ApJ, 781, L31

Soto, M., Rich, R. M., \& Kuijken, K. 2007, ApJ, 665, L31

Thomas, D., Maraston, C., Shawinsky, K., Sarzi, M., \& Silk, J. 2010, MNRAS, 404, 1775

Uttenthaler, S., Schultheis, M., Nataf, D. M., et al. 2012, A\&A, 546, A57

van Dokkum, P., Leja, J., Nelson, E. J., et al. 2013, ApJ, 771, L35

Vásquez, S., Zoccali, M., Hill, V., et al. 2013, A\&A, 555, A91

Zhao, H., Spergel, D. N., \& Rich, R. M. 1994, AJ, 108, 2154

Zoccali, M., Lecureur, A., Barbuy, B., et al. 2006, A\&A, 457, L1

Zoccali, M., Hill, V., Lecureur, A., et al. 2008, A\&A, 486, 177

Zoccali, M., Gonzalez, O. A., Vasquez, S., et al. 2014, A\&A, 562, A66 